\title{
L'apprentissage comme processus social et démocratique chez John Dewey
}

Learning as a social and democratic process in John Dewey

El aprendizaje como proceso social y democrático en John Dewey

Sarah M. Stitzlein

Traducteur : Hélène Bréant

\section{(2) OpenEdition}

Journals

Édition électronique

URL : https://journals.openedition.org/ries/7131

DOI : $10.4000 /$ ries.7131

ISSN : 2261-4265

Éditeur

France Education international

Édition imprimée

Date de publication : 1 décembre 2018

Pagination : 117-125

ISBN : 978-2-85420-620-3

ISSN : $1254-4590$

Référence électronique

Sarah M. Stitzlein, "L'apprentissage comme processus social et démocratique chez John Dewey », Revue internationale d'éducation de Sèvres [En ligne], 79 | décembre 2018, mis en ligne le 01 décembre 2020, consulté le 25 juin 2021. URL : http://journals.openedition.org/ries/7131 ; DOI : https://doi.org/ $10.4000 /$ ries. 7131 


\title{
L'apprentissage comme processus social et démocratique chez John Dewey ${ }^{1}$
}

\author{
Sarah M. Stitzlein \\ Université de Cincinnati
}

\section{LE CONTEXTE HISTORIQUE DES IDÉES DE DEWEY}

John Dewey est indubitablement l'un de « ceux qui ont façonné l'éducation ». Auteur prolifique tout au long de sa vie (1859-1952), son œuvre a influencé les théories et pratiques éducatives aux États-Unis, son pays d'origine, mais aussi dans bien d'autres régions du monde. Ses travaux de recherche et ses écrits ont suscité des progrès considérables dans les champs de la philosophie, de la psychologie, de la théorie politique et de l'éducation. Parmi ses publications les plus remarquables, qui ont relié ses idées en matière éducative à des concepts importants au sein de ces disciplines, figurent les ouvrages suivants : L'École et la société (1899), Démocratie et éducation (1916), Human Nature and Conduct ${ }^{2}$ (1922) et Le Public et ses problèmes (1927).

S'il a rédigé la plupart de ses livres lorsqu'il enseignait à l'Université du Michigan, à l'Université de Chicago et à l'Université Columbia, son œuvre n'est pas restée confinée aux couloirs des universités. Il a créé en effet l'école-laboratoire de l'Université de Chicago, première structure de ce type, où il a mis ses idées à l'épreuve d'enfants et d'enseignants en situation réelle. Il a été aussi une personnalité publique, un intellectuel engagé auprès de plusieurs organisations : président de l'American Psychological Association, responsable syndical militant auprès de la Fédération américaine des enseignants (American Federation of Teachers) et de l'Association américaine des professeurs d'université (American Association of University Professors), il fut l'une des figures de proue de l'Association nationale pour la promotion des personnes de couleur (National Association for the Advancement of Colored People) et administrateur de la Hull-House ${ }^{3}$. Il a également travaillé avec la communauté des immigrés de Chicago, soutenu le Syndicat américain pour les libertés civiles (American Civil Liberties Union), été nommé au conseil d'administra-

1. Article traduit de l'anglais par Hélène Bréant.

2. Nature humaine et comportement, non traduit. (NdT)

3. Fondée par deux femmes de Chicago en 1889, la maison Hull loge des résidents désireux d'habiter un quartier populaire où vivent de nombreux immigrés. Au fil du temps, la résidence fera office de centre socioculturel, abritera une crèche pour les enfants dont les mères travaillent, ou encore une galerie d'art et des bibliothèques. L'engagement des résidents aura aussi permis des avancées législatives majeures pour les droits des enfants et des femmes, notamment. (NdT) 
tion de Bennington College ${ }^{4}$, pris position notamment pendant les grèves Pullman ${ }^{5}$, soutenu le mouvement en faveur du droit de vote des femmes, etc.

Dans le sillage de la Révolution industrielle, Dewey mène ses travaux en accompagnant l'essor de la méthode scientifique et les nouveaux progrès engrangés sur le plan de la compréhension de l'évolution et de l'écologie humaines. Dewey voit le jour l'année où Darwin publie L'Origine des espèces, et nombre de ses idées nouvelles sur la façon dont l'homme échange avec son environnement - et évolue au fil du temps - ont influencé la vision de Dewey qui concevait les enfants comme des organismes dotés de facultés d'adaptation. Dewey est aussi l'un des fondateurs du pragmatisme américain, et ses conceptions de la vérité et de l'enquête se sont concentrées sur la méthode scientifique, se préoccupant moins d'identifier la vérité objective que de déterminer dans quelle mesure le savoir pouvait améliorer le vivre-ensemble. Cette approche a ouvert les portes de l'ère progressiste qui cherchait à utiliser les enquêtes empiriques pour résoudre les problèmes sociaux. Reflet du travail accompli par Dewey avec les immigrés, les minorités et la classe ouvrière, l'ère progressiste a manifesté une préoccupation croissante pour les enfants, les pauvres, et d'autres franges de la population souvent négligées jusqu'alors.

Dewey fut un pionnier de l'éducation progressiste, vivier d'idées nouvelles ayant trait à la fois à la psychologie des apprentissages et aux approches de l'enseignement, lesquelles devaient concourir à améliorer la vie de tous ainsi qu'à développer et à préserver la démocratie. L'éducation progressiste, qui émerge au tout début du vingtième siècle et coïncide avec les critiques de l'industrialisme et de l'accroissement de la fortune des dirigeants, commence à interroger la moindre importance du travail collectif et de la participation démocratique. L'éducation progressiste bat alors en brèche une conception strictement utilitariste de l'école ainsi que la montée en puissance des tests d'intelligence - auxquels on fait appel de plus en plus fréquemment pour suggérer aux enfants un avenir tout tracé. De nouvelles techniques médicales et de mesure sont utilisées pour tester et garantir les aptitudes des apprenants, afin qu'ils soient triés et orientés vers de futurs emplois et fonctions militaires idoines. Plus insidieusement, les eugénistes ont recours à ces techniques pour tenter d'affirmer la supériorité présumée de certaines races. Dewey identifie les conséquences délétères que ces usages de la science pourraient avoir sur la main d'œuvre, le bonheur des hommes et l'organisation de la société.

Alfred Binet et Edward Thorndike, psychologues contemporains de Dewey, ont essentiellement étudié chaque apprenant en tant qu'individu distinct. Dewey, lui, accorde une attention particulière au contexte social et à son influence sur les apprentissages et les activités. Ses travaux nous offrent donc une compréhension plus naturaliste et holistique de l'apprenant que ceux de ses contemporains. Cette démarche a conduit Dewey à la conclusion suivante : les écoles sont des institutions sociales où les enfants devraient interagir avec autrui, tout comme avec les

4. Bennington College est un établissement privé de l'enseignement supérieur américain dédié à la création et aux disciplines artistiques, où l'agir se trouve au cœur de la pédagogie. (NdT)

5. L'entreprise Pullman fabrique des wagons de chemin de fer près de Chicago. Suite à une restructuration et à des pratiques impopulaires, la grève est votée le 10 mai 1894, suivie d'un boycott des produits Pullman en signe de solidarité - avec le soutien du syndicat des chemins de fer américains et des autorités locales. Le mouvement sera brisé par la force à partir du 4 juillet, non sans heurts. Son ampleur et sa nature en font un jalon de l'histoire syndicale et sociale américaine. (NdT) 
programmes scolaires et le monde qui les entoure. Il plaide pour un apprentissage actif qui façonne l'enfant en vue d'un avenir imprévisible, au lieu de le préparer sur un mode passif à un emploi précis associé à un ensemble spécifique de compétences. Selon lui, cet apprentissage passif ne permet pas à l'enfant d'entrer en relation avec le matériau éducatif ni de faire le lien avec ses expériences ou connaissances antérieures.

Ces points de vue s'opposent à l'idéologie concurrente de l'efficacité sociale qui rassemble la psychologie de Thorndike, les objectifs éducatifs de Franklin Bobbitt et les approches industrielles de Frederick Winslow Taylor. Ces derniers prônaient en effet une conception de la scolarité issue du management industriel, dans laquelle des experts standardiseraient toutes les facettes de l'apprentissage, afin que les enfants soient préparés à des emplois spécifiques en acquérant des compétences et connaissances particulières de façon efficace. Cette approche chirurgicale de l'enseignement déqualifie largement les enseignants et échoue à reconnaître leur savoir professionnel ainsi que la nécessité d'adapter les matériaux à la diversité des enfants et des situations ( $\mathrm{Au}, 2011$ ). L'approche contraire que défend Dewey met l'accent sur le développement des facultés d'adaptation, de résolution de problèmes et d'acquisition de compétences sociales, afin que les enfants réussissent à relever les défis qui les attendent, tout en travaillant ensemble comme autant de citoyens au sein d'une démocratie, plutôt que de se préparer à des emplois ciblés. Dans cette configuration, les enseignants sont des artisans qui réagissent avec discernement aux situations qui se produisent dans leur salle de classe - et plus largement dans le contexte d'apprentissage.

Les perspectives de Dewey s'écartent également des recommandations de la Commission des dix (Committee of $\mathrm{Ten}^{6}$ ) et de la Commission des quinze (Committee of Fifteen) qui cherchaient à définir le programme idoine ainsi que les meilleures approches éducatives pour les enfants et les jeunes adultes américains du $\mathrm{xx}^{\mathrm{e}}$ siècle. Ces deux commissions défendaient les matières traditionnelles, souvent au nom de l'acquisition de savoirs comme une fin en soi plutôt que pour leur lien avec les besoins de l'industrie (Bobbitt) ou avec les expériences de l'enfant en tant que citoyen en devenir (Dewey). Pour Thorndike et Bobbitt, les programmes scolaires émanaient des convictions des experts et correspondaient aux besoins de l'industrie. Pour Dewey en revanche, les programmes scolaires s'articulent autour de l'enfant et les enfants sont eux-mêmes à la fois des sources et des bâtisseurs de savoirs et de programmes.

$\mathrm{Au}$ cours des décennies suivantes, les idées de Dewey se sont heurtées à des vagues d'approbation, de scepticisme et de rejet catégorique. La guerre froide a contribué au marasme du mouvement pour l'éducation progressiste, à mesure que l'on construisait des édifices plus rigides et passifs de connaissances factuelles, jugées nécessaires pour accéder à la domination dans l'arène internationale en matière économique et militaire. Néanmoins le mouvement pour l'école libre, l'apprentissage par l'expérience ou par le volontariat, et d'autres démarches pédagogiques expérientielles et ancrées dans l'esprit démocratique suscitent, à la fin du xx siècle, le retour

6. Mise sur pied en 1891 dans le cadre du Conseil national de l'éducation, cette commission se réunit à l'université de Columbia (NYC) du 9 au 11 novembre 1892 et formule des préconisations en vue d'harmoniser les programmes scolaires du secondaire à l'échelle des États-Unis. À partir de juillet 1893, la Commission des quinze entreprit le même travail d’harmonisation pour les programmes élémentaires. 
en grâce de Dewey, notamment auprès des praticiens - certains décideurs politiques restant sceptiques quant à sa philosophie de l'éducation. Plus récemment, l'éducation progressiste a repris de la vigueur et s'inscrit en tension avec "l'enseignement en vue de l'examen ». L'approche scientifique de Thorndike, relative à la standardisation de l'apprentissage et à la mesure des connaissances, a subsisté en effet dans le mouvement pro-examens aux États-Unis et ailleurs, alors qu'un grand nombre d'enseignants qui exercent dans ce mouvement continue de se sentir attiré par les approches de Dewey, plus centrées autour de l'enfant.

\section{AU CEUR DE LA CONCEPTION ÉDUCATIVE DE DEWEY : CROISSANCE, ENQUÊTE ET HABITUDES}

La conception de l'éducation de Dewey s'articule autour du concept de croissance, qui décrit des apprenants doués de facultés d'adaptation qui s'engagent dans une enquête scientifique et qui n'ont de cesse de régénérer leurs habitudes. Contrairement aux approches plus standardisées, en vogue chez les scientifiques au début du $\mathrm{xx}^{\mathrm{e}}$ siècle, qui dissociaient les apprenants de leur environnement, Dewey estime que les humains sont autant d'organismes uniques qui jouissent, au sein de leurs environnements sociaux, du meilleur soutien qui soit. Les interactions familiales et démocratiques apportent aux enfants des occasions de s'adapter et de se transformer, avec l'appui de tiers. Elles renforcent aussi les compétences sociales et le cadre dans lequel les enfants apprennent à apprendre. (Dewey, 1916). Ainsi, tandis que certains spécialistes des sciences de l'éducation considéraient que les enfants étaient trop occupés à des jeux futiles et à des activités familiales stériles, Dewey argue que ces enfants sont en fait occupés par un processus important de croissance au sein de ces espaces. C'est à partir de ces expériences personnelles des enfants à la maison et dans la société qu'il commence à forger ses théories éducatives.

La plupart des gens pensent que la croissance doit se conclure par un point final ou par quelque marque attestant qu'un apprentissage réussi est arrivé à son terme. Dewey retourne cette idée en expliquant que la croissance peut être considérée comme une fin en soi. La croissance est intéressante parce qu'elle nous porte d'une expérience à la suivante, nous offrant ce faisant davantage d'occasions d'apprendre. Ou, pour le dire avec Dewey (1916) :

Puisqu'il n'y a rien à quoi la croissance soit relative sinon plus de croissance, il n'y a rien à quoi l'éducation doive être subordonnée sinon plus d'éducation ${ }^{7}$.

Il a attiré l'attention sur ces expériences éducatives qui mènent à d'autres expériences enrichissantes, à un apprentissage permanent et à plus de croissance un processus qui se poursuit sans avoir de point final prédéfini. C'est ce que veut dire Dewey lorsqu'il affirme que le processus éducatif n'a d'autre finalité que la continuation de lui-même. Le rôle de l'enseignant est alors de faciliter la croissance. Cela s'écarte des techniques d'efficacité sociale telles que les approches mécaniques

7. Traduction française d'après G. Deladalle, Démocratie et éducation, Paris, L'Âge d'homme, 1983. 
et répétitives qui postulent une finalité spécifique à laquelle chaque enfant devrait parvenir, au lieu de mettre l'accent sur la croissance en tant que telle.

Dewey partage avec Jean-Jacques Rousseau l'éloge de la curiosité naturelle des enfants et de leur capacité à apprendre. Dewey souligne la plasticité des enfants, qui leur permet d'apprendre de leurs expériences et, par conséquent, de se transformer et/ou de changer le monde. Une telle plasticité facilite le développement d'habitudes qui permettent aux apprenants de fonctionner correctement. Dewey a puisé dans les idées de Darwin sur l'adaptation et les a étendues en expliquant comment la réflexion intelligente sert de guide à l'adaptation. Dewey en conclut que lorsque nous apprenons en faisant des essais, nous serons plus tard capables de modifier nos actions dans des situations inédites. Ainsi, en apprenant à nous adapter, nous apprenons à apprendre.

La plupart des gens entendent les habitudes comme un comportement routinier que nous répétons généralement sans aucune réflexion consciente - Dewey rétorque que les habitudes sont des propensions à agir. Cette action nous permet de nous impliquer dans le monde, d'apprendre de lui, de nous adapter à lui au fur et à mesure de notre croissance. Les habitudes sont dès lors des éléments essentiels dans la conception éducative de Dewey. Les habitudes sont des manières d'être et d'agir, des sensibilités et des tempéraments qui nous permettent de vivre notre quotidien sereinement. Elles commencent par des élans qui nous incitent naturellement à agir. À travers nos expériences des normes culturelles et, au-delà, du monde, ces élans se cumulent et se font habitudes. Dewey (1916) l'explique ainsi :

Toute habitude marque une inclination - une préférence active et un choix des conditions nécessaires à sa pratique. Une habitude n'attend pas - tel M. Micawber qu'un stimulus se présente pour entrer en jeu : elle cherche activement des occasions de passer pleinement à l'action ${ }^{8}$.

Ainsi, les habitudes ne sont donc pas de simples attitudes " par défaut »; il faut en faire l'expérience.

Mais les habitudes ne se soucient pas uniquement d'agir, elles sont aussi reliées à la pensée. Les habitudes " font tout le travail de perception, de reconnaissance, d'imagination, de remémoration, de jugement, de conceptualisation et de raisonnement ${ }^{9}$ "(Dewey, 1922). Les habitudes nous aident à insuffler du sens au monde auquel nous sommes confrontés, façonnant ce faisant nos idées. Puis les habitudes traduisent concrètement nos pensées en les mettant à l'épreuve du monde. Lorsque nous enquêtons sur leurs effets et que nous réfléchissons à leur propos en tant que partie intégrante du processus d'apprentissage, nous sommes incités à ajuster nos habitudes actuelles ou à en développer de nouvelles, afin de mieux satisfaire à nos besoins. Bien plus qu'une pure routine, nos habitudes sont donc étroitement liées à l'intelligence et sont un vecteur d'apprentissage et de croissance.

C'est avant tout à l'école que nous cultivons des habitudes lorsque nous regardons, imitons et interagissons avec nos enseignants et nos pairs. L'enseignant devrait aider les élèves à utiliser le processus de l'enquête scientifique pour les guider

8. Traduction française : Démocratie et éducation, suivi d’Expérience et éducation, Armand Colin, 2018. (NdT)

9. Traduction française de L. Quéré (2014) : "Le modèle esthétique de la communication de John Dewey, Occasional Paper 19, Paris, Institut Marcel Mauss - CEMS. (NdT) 
dans la formation et l'évolution de leurs habitudes. Ainsi, plutôt que de se contenter de dispenser des savoirs ou des habiletés, les enseignants devraient façonner des environnements où les enfants seront enclins à apprendre par eux-mêmes. Cela requiert une relation étroite entre l'élève et l'enseignant, si bien que ce dernier aura une bonne compréhension de l'environnement de l'élève, de ses intérêts, et bien davantage. En somme, l'enseignant travaille à offrir un environnement et à guider l'enquête, afin que les élèves développement des habitudes flexibles qui conduisent à leur croissance et à leur épanouissement.

\section{L'IMPACT DE DEWEY À TRAVERS LE TEMPS ET LE MONDE}

C'est dans son pays d'origine, les États-Unis, que l'impact de John Dewey a été le plus significatif. Au cours de sa carrière, il a fait cependant plusieurs voyages à l'étranger au cours desquels son impact a été considérable et, dans bien des cas, a persisté pendant des décennies et jusqu'à nos jours. Ses idées sont notamment autant d'outils de démocratisation et de modernisation dans des pays encore jeunes ou en développement.

Dewey a longtemps séjourné en Asie orientale. Il a passé deux mois à enseigner à l'Université de Tokyo en 1919. De 1919 à 1921, il vit en Chine, où son impact sur le pays a été très prononcé. Au départ, son travail a été utilisé pour soutenir le mouvement chinois dit de la Nouvelle Culture qui fleurit du mitan des années 1910 à la fin des années 1920. Les Chinois en quête d'occidentalisation et de démocratisation ont embrassé le pragmatisme de Dewey et ses théories politiques. À son retour aux États-Unis, Dewey rapporte avec lui ses observations sur la Chine, dans l'espoir de contribuer à la compréhension interculturelle. En 1926, Dewey, Hu Shih, Paul Monroe et le professeur Kuo Ping-wen fondent à New York The China Institute in America, une institution à caractère éducatif et culturel.

Aujourd'hui encore, les idées de Dewey fascinent nombre d'universitaires chinois, qui s'emploient à les diffuser. Tout récemment, les très nombreux écrits de Dewey ont été traduits en mandarin, grâce aux efforts de l'université East China Normal University, du Dewey Center de la Fudan University à Shanghaï et du Pragmatism Project qu'abrite l'Université normale de Pékin. Chacune de ces universités continue d'enseigner Dewey.

Dewey étendit également son influence à l'Europe orientale et à l'Asie. En 1924, peu après que la Turquie a conquis son indépendance, Dewey est invité par le nouveau président à introduire les idéaux démocratiques dans son pays. Il travaille avec les dirigeants turcs pour imaginer la manière dont l'enseignement public pourrait jouer un rôle central dans la constitution et la croissance de leur démocratie. En 1928, Dewey se rend en Union soviétique. Si l'espoir caractérise ses premières réactions devant l'état d'avancement de la réforme de l'éducation en Union soviétique, le système et le leadership politique le décourageront au fil du temps - il les considèrera en effet comme incompatibles avec sa conception de la démocratie. Beaucoup plus récemment, un Centre d'études sur Dewey (Dewey Studies Center) a été créé en Slovaquie. Quoique les conceptions de Dewey aient reçu un accueil plus sceptique en Slovaquie et en République tchèque, quelques universitaires de ce 
Centre contribuent à transmettre son message centré sur les interrelations et l'apprentissage social - ce qui pourrait avoir une certaine importance pour vivre dans l'Europe centrale et orientale contemporaine.

En 1934, Dewey part en Afrique du Sud pour une série de conférences et pour visiter des écoles. Depuis, des tenants de deux courants théoriques de l'éducation se sont saisis de ses travaux : la perspective conservatrice, largement fondée sur "l'éducation nationale-chrétienne ${ }^{10}$ ", et la perspective du "développement curriculaire alternatif »(Alternative Curriculum Development). Ce dernier, plus proche des conceptions de Dewey, met l'accent sur l'interaction et les réformes sociales. Plus récemment, en Afrique du Sud, les idées de Dewey ont présidé au développement de l'apprentissage par le volontariat, qui fait la synthèse entre les conceptions de Dewey relatives à l'apprentissage par l'expérience et des activités collectives hors du cadre scolaire, au sein de la communauté locale.

En 1937, Dewey s'envole pour le Mexique, où il va présider la commission d'enquête internationale sur Léon Trotski ${ }^{11}$, quinze ans environ après l'introduction dans ce pays des conceptions éducatives de Dewey par deux de ses anciens étudiants au Teachers College, de l'université Columbia. Le Mexique s'efforce alors de se remettre de la violence de la révolution; le pays cherche à créer des écoles qui rassemblent des groupes culturellement hétérogènes ainsi qu'à étendre l'enseignement aux régions rurales reculées. Ces anciens élèves de Dewey s'emploient à tisser des liens étroits entre les écoles fraîchement ouvertes et leurs communautés.

\section{L'UTILITÉ DES IDÉES DE DEWEY POUR LE PRÉSENT}

Les idées de Dewey restent d'actualité pour de nombreux enseignants d'aujourd'hui ; elles peuvent être mises au service de l'éducation dans certains combats actuels. Tout d'abord, les notions de croissance, d'enquête et de formation d'habitudes restent des sources d'inspiration pour la pédagogie expérientielle, notamment pour la pédagogie du plein air où les élèves sont confrontés en permanence à de nouvelles situations qui requièrent réflexion et adaptation pour réussir. Même dans des contextes plus classiques de salles de classe, l'apprentissage par l'expérience appelle une implication tangible au contact d'objets et de matériaux du monde réel liés à la vie personnelle de chacun, et ce afin de développer la compréhension et de forger la croissance, si bien que l'apprenant peut aller d'expérience en expérience. L'importance que Dewey accorde à l'éducation expérientielle comme processus actif s'oppose au rôle central de la mémorisation en vue d'examens standardisés devenus monnaie courante au cours des dernières décennies, aux États-Unis et ailleurs. Alors que cette tendance inflationniste aux évaluations semble refluer, du moins aux États-

10. Christian-national Education (CNE) : doctrine de l'État boer inspirée du calvinisme genevois et matinée d'influences nationales-socialistes, la CNE oppose notamment à la théorie de l'évolution celle de la prédestination. $(N d T)$

11. Lorsque, depuis son exil au Mexique, Trotski appelle à la création d'une commission d'enquête internationale pour montrer le caractère mensonger des accusations portées contre lui lors des procès de Moscou, de nombreux intellectuels de gauche refusent de s'engager. Dewey, lui, accepte sans hésiter. (NdIR, d'après Leur morale et la nôtre, John Dewey, Léon Trotski, préface d’É. Hache, éd. La Découverte (Les empêcheurs de penser en rond), 2014. 
Unis, les travaux de Dewey pourraient peut-être être renouvelés pour nous aider à comprendre l'importance de l'expérimentation, de l'apprentissage expérientiel et des travaux collectifs en classe. Les travaux de Dewey pourraient aussi être étendus pour permettre des avancées en matière d'apprentissage fondé sur la résolution de problèmes ou de pédagogie de projet - deux démarches prometteuses pour impliquer les enfants et les préparer à résoudre des problèmes sociaux plus importants, que ce soit à l'école ou après la fin de leurs études. Les travaux de groupes étant de plus en plus appréciés à l'école comme dans le monde du travail, les idées de Dewey relatives à la circulation des connaissances entre participants et à la manière dont on peut développer collectivement le savoir méritent d'être rappelées.

En outre, les conceptions de Dewey sur la croissance et l'adaptabilité recèlent des liens importants avec les travaux de chercheurs contemporains tels que Carol Dweck et Paul Tough. Ceux-ci s'emploient à déterminer, à travers leurs recherches, quelles caractéristiques (avoir un état d'esprit orienté vers la croissance, par exemple) suscitent l'apprentissage tout au long de la vie et la réussite sur le plan académique. À l'instar de la thèse de Dewey sur la formation des habitudes et le changement, les travaux de Dweck démontrent que l'état d'esprit de chacun est malléable et qu'un apprenant peut être disposé à cultiver de nouvelles habitudes.

Par ailleurs, de nombreux pays du monde sont confrontés à une offensive néolibérale en faveur de la privatisation de l'enseignement. La philosophie de l'éducation de Dewey trace des pistes pour réagir à cette offensive ${ }^{12}$. Dans le cadre de ces tentatives de privatisation, certains réformateurs se tournent de plus en plus vers l'enseignement à distance comme vecteur moins cher et plus efficace pour éduquer les enfants. Si l'enseignement à distance peut permettre aux écoles de mieux adapter leur cursus aux intérêts singuliers de chaque enfant - ce que Dewey ne renierait pas ; il s'inquièterait cependant de ce que cette technique échoue à reconnaître que l'éducation devrait être un effort de nature profondément sociale, au cours duquel les enfants s'impliquent ensemble dans l'apprentissage et la résolution de problèmes. Trop souvent, l'enfant assis devant un ordinateur n'interagit pas avec les autres apprenants, avec l'environnement physique ni avec les problèmes sociaux. De même, insister sur les résultats privés de l'éducation se heurterait à l'idéal de Dewey, pour qui le but de l'éducation est le bien commun. Il a en effet proposé un sens très fort de la participation publique à l'éducation et soigneusement consigné les bienfaits publics de l'éducation en tant qu'institution de la démocratie. Il appelait à constituer des publics qui reconnaissent les écoles comme un bien public et revendiquent ce point de vue.

Enfin, nombreux sont les pays où les syndicats d'enseignants sont en lutte et où le rôle des syndicats et le professionnalisme des enseignants sont de plus en plus souvent remis en cause. Ces conceptions sont exacerbées par l'extension des marchés libres néolibéraux qui considèrent les syndicats comme autant d'obstacles à l'efficacité et à l'innovation. De surcroît, les progrès de l'informatique suggèrent que certains rôles de l'enseignant pourraient être remplacés par des ordinateurs installés dans la classe. Dewey était un ardent défenseur des syndicats d'enseignants et du professionnalisme de ces derniers. Il expliquait qu'il s'agit de professionnels qui s'en-

12. C'est justement ainsi que j'ai convoqué Dewey dans mon dernier livre, voir Stitzlein S. M. (2017). 
gagent sur la voie de l'enquête et de l'expérimentation afin d'améliorer leur pratique, au lieu de se contenter d'appliquer des palettes spécifiques de compétences prédéfinies ou de suivre à la lettre un manuel à l'usage des enseignants. Évidemment, ces points de vue auront des conséquences sur une formation des enseignants de qualité, en lieu et place d'une formation expéditive des enseignants empruntant des détours de plus en plus populaires, tels que l'initiative Teach for America ${ }^{13}$. Les nombreux écrits de Dewey fournissent une sémantique et un cadre permettant de réagir à certaines de ces attaques contre la formation des enseignants, leur professionnalisme et leurs syndicats.

En un mot, l'impact de Dewey sur l'éducation fut et demeure considérable. Il a introduit entre les murs des écoles des idées originales dans les champs de la théorie politique, de la psychologie et de la philosophie. Et si les applications de ses travaux ont connu des hauts et des bas, il y a fort à parier que ses concepts et idées conserveront toute leur utilité dans les entreprises éducatives à venir.

\section{RÉFÉRENCES BIBLIOGRAPHIQUES}

AU W. (2011) : Teaching under the new Taylorism: High stakes testing and the standardization of the 21st century curriculum, Journal of Curriculum Studies, 43(1), p. 25-45.

DEWEY J. (1966[1916]) : Democracy and Education, New York, The Free Press.

DEWEY J. (2002[1922]) : Human Nature and Conduct (Mineola, NY, Dover).

KNIGHT ABOWITZ K. (2014) : Publics for Public Schools: Legtimacy, Democracy, and Leadership, New York, Routledge.

STITZLEIN S. M. (2017) : American Public Education and the Responsibility of Its Citizens: Supporting Democracy in the Age of Accountability, New York, Oxford University Press.

13. Teach for America est une organisation à but non lucratif créée en 1989 pour pallier la pénurie d'enseignants dans les écoles publiques et lutter contre les inégalités sociales, en envoyant de jeunes diplômés de l'enseignement supérieur enseigner pendant deux ans auprès de publics défavorisés. ( $N d T$ ) 
